\title{
Regularizations: Different Prescriptions for Identical Situations
}

\author{
E. Gambin*, C. O. Lobo*, G. Dallabona**, and O. A. Battistel* \\ * Dept. of Physics, Universidade Federal de Santa Maria \\ P.O. Box 5043, 97119-900, Santa Maria, RS, Brazil \\ Instituto de Física Teórica,Rua Pamplona, 145, 01405-900, São Paulo, SP, Brasil
}

Received on 10 December, 2004

\begin{abstract}
We present a discussion where the choice of the regularization procedure and the routing for the internal lines momenta are put at the same level of arbitrariness in the analysis of Ward identities involving simple and well-known problems in quantum field theory. They are the complex self-interacting scalar field and two simple models where the scalar-vector-vector and axial-vector-vector process are pertinent. We show that, in all these problems, the conditions to symmetry relations preservation are put in terms of the same combination of divergent Feynman integrals, which are evaluated in the context of a very general calculational strategy, concerning the manipulations and calculations involving divergences. Within the adopted strategy, all the arbitrariness intrinsic to the problem are still maintained in the final results and, consequently, a perfect map can be obtained with the corresponding results of traditional regularization techniques. We show that, when we require an universal interpretation for the arbitrariness involved, in order to get consistency with all stated physical constraints, a strong condition is imposed for regularizations which automatically eliminates the ambiguities associated to the routing of the internal lines momenta of loops. The conclusion is clean and sound: the association between ambiguities and unavoidable symmetry violations in Ward identities cannot be maintained if an unique prescription is required for identical situations in the evaluation of divergent physical amplitudes.
\end{abstract}

\section{INTRODUCTION}

The first step in the construction of a quantum field theory (QFT) is the building of the corresponding Lagrangian. The symmetry content, which means invariance under a set of transformations, implies in definite relations among the Green's functions of the theory. Frequently, these symmetry relations or Ward identities involve the evaluation of divergent Green's functions. It is crucial for the renormalization of the theory or for the derivation of low-energy theorems that such relations are preserved at any order of the perturbative evaluation. The role of the regularization technique can be decisive in the verification of the symmetry relations. Since that, in spite of the divergences, they must be verified case by case, there is a self consistent aspect involved in these discussions. In one hand a consistent technique to handle the divergences is the one that does not lead to undesirable features like ambiguities and/or symmetry relations violations, which means to destroy the predictive power of the corresponding QFT or to spoil the renormalizability of the theory. On the other hand, for the significance of the theory in the perturbative approach we need to verify the symmetry relations which means to adopt a philosophy to handle the divergences in a consistent way. So, when we evaluate a set of divergent Green's functions using a particular regularization procedure and a certain symmetry relation involving them is not verified satisfied, in principle, we cannot conclude in a positive way if the violation is a consequence of the inconsistency of the employed method or if we are facing an unavoidable phenomenon of symmetry breaking like the triangle anomalies seem to be in QFT. Strictly speaking, we can only classify a violation of symmetry as an anomaly if we are convinced that does not exist and must not exist a technique that is capable to avoid the violation. The eventual violating terms cannot be dependent on the regularization technique. The same reasoning line can be applied to the symmetry preserving relations. We, in prin- ciple, can only convince ourselves that a symmetry relation is preserved if the verification is clearly not dependent on the specific aspects of the adopted regularization method. In order to allow the desirable accommodation of both situations, violations and preservations of symmetries, no role can be played by the regularization scheme or equivalent technique. The ideal situation is to perform the necessary calculations without the explicit use of a regularization. In certain sense the conclusion above is obvious. Since the choice of auxiliary tools is arbitrary, no dependence on the specific aspects involved is accepted due to the fact that this implies ambiguity. The real situation of the problem, however, is somehow different. In the verification of the preservation of symmetries in perturbative evaluation, the explicit use of regularization philosophies is almost always adopted. In the establishment of the desirable violations of symmetry relations (anomalies), specific perturbative aspects (divergences and ambiguities) play a crucial role. The situation can be summarized in a simple way. In all the situations where the Dimensional Regularization (DR) [1] technique can be applied we certainly adopt it. The method provides a prescription that is well-succeeded in the avoidance of ambiguities and, simultaneously, symmetry relations violations. However, as is well-known, the DR technique is not general. There are situations where we cannot apply it within an universal prescription characterizing the method. We refer to the situations where the $\gamma_{5}$ Dirac matrix (or the totally antisymmetric tensor $\varepsilon_{\alpha \beta \mu \nu}$ ), or their similars in other space-time dimensions, play an important role. Since such objects and their algebra are not extendable, a particular prescription becomes necessary in each previously chosen dimension. Given the fact that such prescriptions are not unique, new types of ambiguities may arise in the perturbative calculations, associated to the employed regularization technique, which introduces more undesirable ingredients in a problem complicated by self. In consequence, we are forced to have recourse to other methods which may not be consistent con- 
cerning the avoidance of ambiguities and symmetry relations violations. In other words, to treat a problem where the DR cannot be applied, we adopt a procedure that, if applied to treat a problem where the consistent results are achieved by the DR, may lead to unacceptable results. This is precisely the situation of the axial-vector-vector $(A V V)$ triangle anomaly [2] - [6]. Due to the presence of an odd number of $\gamma_{5}$ Dirac matrix, we are prevented to use in a natural way the DR. As a consequence, ingredients which are automatically excluded within the context of DR are called to play a decisive role in the evaluations which is the case of the internal momenta ambiguities.

In the present work we discuss questions related to the analysis of Ward identities involving divergent amplitudes. For this purpose, we select three simple but representative models and generate the corresponding symmetry relations. The main aspect is the fact that we can put all the considered Ward identities in terms of the same condition. After this, we use a very general calculational strategy [7], concerning the divergences manipulations and calculations, in order to evaluate the divergent Feynman integrals involved. In the adopted method, all the arbitrariness intrinsic to the problem are preserved and a map with the DR results and with those produced by the surface's term analysis is always possible. These two maps, however, are obtained through conflicting interpretations for the involved arbitrariness $[8,9]$. We show that, when we require an unique interpretation for the indefinitions, interesting questions about the perturbative origin of the $A V V$ anomaly emerge [8].

The work is organized as follows. In the section II we derive, in detail, a Ward identity for the self-interacting complex scalar field. In the section III and IV, we consider a simple model to the scalar-vector-vector $(S V V)$ and $A V V$ process, respectively, and their associated symmetry relations. The calculational strategy, used in the treatment of divergent Feynman integrals, is introduced in the section $\mathrm{V}$, whose results are substituted in all the obtained Ward identities in the section VI. In the section VII we use the general results obtained from our analysis in order to recover the traditional treatment for the $A V V$ triangle anomaly. Finally, in the section VIII we present our final remarks and conclusions.

\section{THE COMPLEX SCALAR FIELD}

Perhaps the most simple QFT where a symmetry relation can be stated is the $\lambda \phi^{4}$ theory. A Ward identity can be easily constructed for the complex scalar field due to the existence of a conserved vector current. In this section, we follow in a closely related way the ref.[10] in order to state the symmetry relation. The corresponding Lagrangian can be written as

$$
\mathcal{L}=\left(\partial_{\mu} \phi^{*}\right)\left(\partial^{\mu} \phi\right)-\mu^{2}\left(\phi \phi^{*}\right)-\lambda\left(\phi \phi^{*}\right)^{2},
$$

where $\mu$ is the mass of the scalar field and $\lambda$ is the coupling constant. The above Lagrangian is invariant under $U(1)$ transformations

$$
\phi \rightarrow \phi^{\prime}=e^{i \alpha \cdot T} \phi
$$

where $\alpha$ is a constant (independent of $x$ ) and $T$ is a c-number. Such invariance gives raise to the conserved vector current

$$
J_{\mu}=i\left[\left(\partial_{\mu} \phi^{*}\right) \phi-\left(\partial_{\mu} \phi\right) \phi^{*}\right]
$$

The complex scalar field satisfies the following canonical commutation relation

$$
\left[\partial_{0} \phi^{\dagger}(\vec{x}, t), \phi\left(\overrightarrow{x^{\prime}}, t\right)\right]=-i \delta^{3}\left(\vec{x}-\overrightarrow{x^{\prime}}\right),
$$

which leads us to the following commutators involving the fields and currents

$$
\begin{aligned}
{\left[J_{0}(\vec{x}, t), \phi\left(\overrightarrow{x^{\prime}}, t\right)\right] } & =i\left[\partial_{0} \phi^{\dagger}(\vec{x}, t), \phi\left(\overrightarrow{x^{\prime}}, t\right)\right] \phi(\vec{x}, t) \\
& =\delta^{3}\left(\vec{x}-\overrightarrow{x^{\prime}}\right) \phi(\vec{x}, t) \\
{\left[J_{0}(\vec{x}, t), \phi^{\dagger}\left(\overrightarrow{x^{\prime}}, t\right)\right] } & =-\delta^{3}\left(\vec{x}-\vec{x}^{\prime}\right) \phi^{\dagger}(\vec{x}, t) .
\end{aligned}
$$

With these ingredients it is possible to consider a process involving a vector current and two scalar fields and the corresponding symmetry relation. For this purpose let us consider the Green's function

$G_{\mu}(p, q)=\int d^{4} x d^{4} y e^{(-i q \cdot x-i q \cdot y)}<0\left|T\left(J_{\mu}(x) \phi(y) \phi^{\dagger}(0)\right)\right| 0>$.

In order to get a symmetry relation we take the fourdivergence in both sides of the equation above and, in the integrand, use standard manipulations of the current algebra

$$
\partial_{x}^{\mu}\left[\left(T\left(J_{\mu}(x) O(y)\right)\right]=T\left(\partial^{\mu} J_{\mu}(x) O(y)\right)+\left[J_{0}(x), O(y)\right] \delta\left(x_{0}-y_{0}\right) .\right.
$$

After this step we get

$$
\begin{aligned}
& q_{\mu} G^{\mu}(p, q) \\
& =-i \int d^{4} x d^{4} y e^{-i q \cdot x-i p \cdot y} \partial_{\mu}<0\left|T\left(J_{\mu}(x) \phi(y) \phi^{\dagger}(0)\right)\right| 0> \\
& =-i \int d^{4} x d^{4} y e^{-i q \cdot x-i p \cdot y}\left\{<0\left|T\left(\partial_{\mu} J_{\mu}(x) \phi(y) \phi^{\dagger}(0)\right)\right| 0>\right. \\
& +<0\left|T\left(\left[J_{0}(x), \phi(y)\right] \delta\left(x_{0}-y_{0}\right) \phi^{\dagger}(0)\right)\right| 0> \\
& \left.+<0\left|T\left(\left[J_{0}(x), \phi^{\dagger}(0)\right] \delta\left(x_{0}\right) \phi(y)\right)\right| 0>\right\} .
\end{aligned}
$$

Given the conservation of the vector current the first term in the equation above vanishes. Using then the commutation relations (5) and (6) we are left with

$$
\begin{aligned}
q_{\mu} G^{\mu}(p, q) & =-i \int d^{4} x d^{4} y e^{-i(q+p) x}<0\left|T\left(\phi(x) \phi^{\dagger}(0)\right)\right| 0> \\
& +i \int d^{4} x d^{4} y e^{-i p y}<0\left|T\left(\phi(0)^{\dagger} \phi(y)\right)\right| 0>\text {. (10) }
\end{aligned}
$$

Next, we can identify the two terms on the right hand side as propagators of the scalar field

$$
\Delta(p)=\int d^{4} x e^{-i p x}<0\left|T\left(\phi(x) \phi^{\dagger}(0)\right)\right| 0>,
$$

and then write

$$
-i q_{\mu} G^{\mu}(p, q)=\Delta(p+q)-\Delta(p),
$$


which is the vector-current Ward identity. The equation above holds for the corresponding renormalized quantities due to the fact that the conserved current $J_{\mu}(x)$ is not renormalized as a composite operator [4]. It is then easy to state the corresponding one-loop version for the eq.(12). For this purpose we define the amputed Green's functions, in terms of the renormalizable quantities present in the eq.(12); in the following way [10]

$$
\Gamma_{\mu}(p, q)=\left[i \Delta^{R}(p+q)\right]^{-1} G_{\mu}^{R}(p, q)\left[i \Delta^{R}(p)\right]^{-1},
$$

where the one-loop renormalized propagator is given by

$$
\Delta^{R}(p)^{-1}=p^{2}-\mu^{2}-\tilde{\Sigma}\left(p^{2}\right),
$$

and $\tilde{\Sigma}\left(p^{2}\right)$ is the one particle irreducible (1PI) self-energy. Then the Ward identity (12) assumes the simple form

$$
i q^{\mu} \Gamma_{\mu}(p, q)=\left[(p+q)^{2}-\mu^{2}-\tilde{\Sigma}(p+q)\right]-\left[p^{2}-\mu^{2}-\tilde{\Sigma}(p] .\right.
$$

Let us now consider the explicit evaluation at tree level and next at the one-loop level. For this purpose we start by considering the coupling among the conserved vector current with the two scalar lines. The corresponding vertex is given by

$$
\begin{aligned}
\frac{i \delta^{3} \mathcal{L}_{I}}{\delta J_{\mu} \delta \phi \delta \phi^{*}} & =i^{2}\left[i(p+q)_{\mu}+i p_{\mu}\right] \\
& =-i(2 p+q)_{\mu} .
\end{aligned}
$$

The tree level contribution, diagrammatically represented in the Fig. 1, can be easily evaluated as

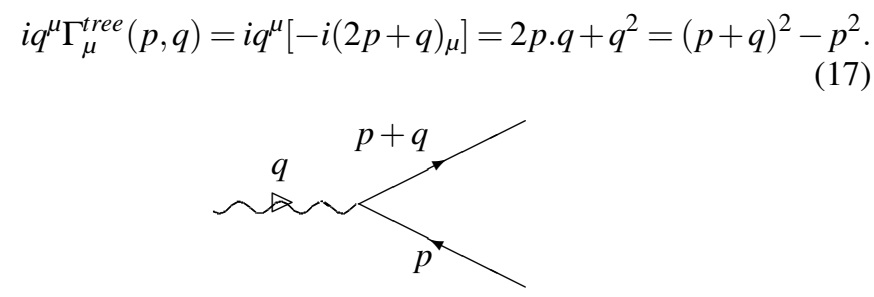

Figure 1: Diagrammatic representation for the tree level contribution.
The comparison with the expression (15) reveals that, at the tree level, the identity (12) is preserved. Let us now consider the one-loop level, diagrammatically represented in the Fig. 2 and Fig. 3.

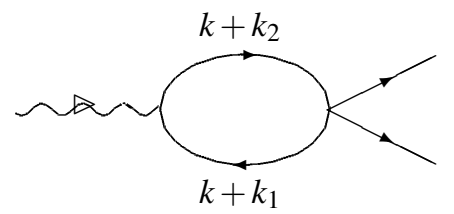

Figure 2: Diagrammatic representation for the one-loop contribution to the vertex correction.

The first two diagrams in the Fig. 3 require the evaluation of the self-energy at the one-loop level, which is given by

$$
-i \Sigma(p)=-i \frac{\lambda}{2} \int \frac{d^{4} k}{(2 \pi)^{4}} \frac{i}{(k+l)^{2}-\mu^{2}},
$$

where we have adopted an arbitrary routing for the internal line momentum of the loop. The one-loop renormalization implies in the addition of the counterterm's diagrams, Fig. 3.

The contribution of the first two diagrams to the $\Gamma_{\mu}(p, q)$ vertex function can be written as

$$
i q^{\mu} \Gamma_{\mu}(p, q)=i q^{\mu}\left\{(-i)(2 p+q)_{\mu} \frac{i}{(p+q)^{2}-\mu^{2}}[\Sigma(p+q)-\Sigma(0)]\right\}
$$

which vanishes identically due to the independence of the external momentum of the scalar one-loop self-energy. So, we are left only with the contribution of the diagram in the Fig. 2. The contribution for the symmetry relation is given by

$$
i q^{\mu} \Gamma_{\mu}^{1 \text { loop }}(p, q)=i q^{\mu}\left\{\int \frac{d^{4} k}{(2 \pi)^{4}}(i \lambda) \frac{i}{\left[\left(k+k_{1}\right)^{2}-\mu^{2}\right]}(-i)\left(2 k+k_{1}+k_{2}\right)_{\mu} \frac{i}{\left[\left(k+k_{2}\right)^{2}-\mu^{2}\right]}\right\},
$$

which means that

$$
i q^{\mu} \Gamma_{\mu}^{1 \text { loop }}(p, q)=i \lambda\left(k_{1}-k_{2}\right)^{\mu}\left\{\int \frac{d^{4} k}{(2 \pi)^{4}} \frac{2 k_{\mu}+\left(k_{1}+k_{2}\right)_{\mu}}{\left[\left(k+k_{1}\right)^{2}-\mu^{2}\right]\left[\left(k+k_{2}\right)^{2}-\mu^{2}\right]}\right\}
$$



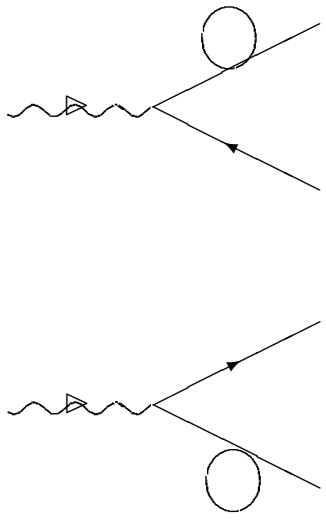
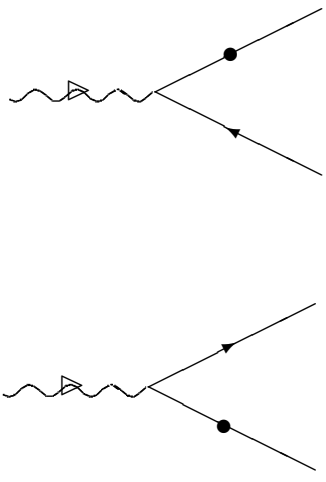

Figure 3: Diagrammatic representation for the one-loop corrections and their counterterms diagrams.

or

$$
i q^{\mu} \Gamma_{\mu}^{1 \text { loop }}(p, q)=i \lambda\left(k_{1}-k_{2}\right)^{\mu}\left(\Delta I_{\mu}\right) .
$$

We have arrived at the main point of this section. Given the fact that the one-loop scalar self-energy does not have a finite part, the Ward identity is satisfied by the tree level contribution. This implies that all the contribution of the one-loop level must cancel. Since two diagrams cancel two others it remains only the contribution of one diagram which must identically vanish by itself. In the corresponding expression, two divergent integrals are involved with a degree of divergence linear and logarithmic. Independent on the details involved, which we will discuss later, it is clear that if the value for the specific combination of integrals

$$
\Delta I_{\mu}=2\left(I_{2}\right)_{\mu}+\left(k_{1}+k_{2}\right)_{\mu}\left(I_{2}\right),
$$

where we have introduced the definitions,

$$
\left(I_{2} ; I_{2}^{\mu}\right)=\int \frac{d^{4} k}{(2 \pi)^{4}} \frac{\left(1 ; k^{\mu}\right)}{\left[\left(k+k_{1}\right)^{2}-m^{2}\right]\left[\left(k+k_{2}\right)^{2}-m^{2}\right]},
$$

does not vanish, the Ward identity which we have stated will be violated. Due to the divergences the evaluation of the expression (23) requires the adoption of a regularization technique or an equivalent philosophy. Before such discussions let us state other kinds of Ward identities.

\section{III. $S \rightarrow V V$ PROCESS}

Let us now consider a theory where the scalar and the vector fermionic densities are coupled to a scalar and a vector field, respectively. In this section we perform the discussions in a similar way to that of the ref.[11]. The interaction Lagrangian can be written as

$$
\mathcal{L}_{I}=i G_{s}(\bar{\Psi} \Psi) \phi-G_{v}\left(\bar{\Psi} \gamma^{\mu} \Psi\right) A_{\mu}
$$

where $\Psi$ is a massive $\frac{1}{2}$ spin fermion, $\phi$ is a scalar field and $A_{\mu}$ a vector field. The fermionic vector current obeys

$$
\partial_{\mu} V^{\mu}=\partial_{\mu}\left(\bar{\Psi} \gamma^{\mu} \Psi\right)=0
$$

i.e., due to the presence of only one specie of massive fermion the scalar and vector currents are not connected. So, if we define the $S \rightarrow V V$ Green's function

$$
\begin{aligned}
& T_{\mu v}^{S \rightarrow V V}\left(p, p^{\prime} ; q\right)=i \int d^{4} x_{1} d^{4} x_{2} e^{i p x_{1}+i p^{\prime} x_{2}} \\
& \times<0\left|T\left(V_{\mu}\left(x_{1}\right) V_{v}\left(x_{2}\right) S_{0}(0)\right)\right| 0>
\end{aligned}
$$

following the standard procedure of the current algebra, we must get the Ward identities

$$
\left\{\begin{aligned}
p^{\mu} T_{\mu v}^{S \rightarrow V V} & =0 \\
p^{\prime \nu} T_{\mu v}^{S \rightarrow V V} & =0
\end{aligned}\right.
$$

The lowest order perturbative contribution for the $S \rightarrow V V$ process is given by the triangle diagram. The summation of the direct and crossed diagrams is required by the Bose final state symmetrization. In the evaluation of these contributions, we will assume the routings of the internal lines as the most general ones. For the direct channel, Fig.4(a), we adopt the internal lines momenta as being related to the external ones 
by their differences as follows

$l_{1}, l_{2}$ and $l_{3}$, satisfying

$$
\left\{\begin{array}{l}
k_{3}-k_{2}=q=p+p^{\prime} \\
k_{3}-k_{1}=p \\
k_{1}-k_{2}=p^{\prime}
\end{array}\right.
$$

$$
\left\{\begin{array}{l}
l_{3}-l_{2}=q=p+p^{\prime} \\
l_{3}-l_{1}=p^{\prime} \\
l_{1}-l_{2}=p
\end{array}\right.
$$

The crossed diagram can be constructed by changing $\mu$ and $v$ and adopting for the internal lines the arbitrary momenta as

$$
T_{\mu \nu}^{S V V}\left(k_{1}, k_{2}, k_{3} ; m\right)=\int \frac{d^{4} k}{(2 \pi)^{4}} \operatorname{Tr}\left\{\hat{1} \frac{1}{\left(\not k+k_{3}\right)-m} \gamma_{\mu} \frac{1}{\left(k+k_{1}\right)-m} \gamma_{v} \frac{1}{\left(k+k_{2}\right)-m}\right\}
$$

(a)

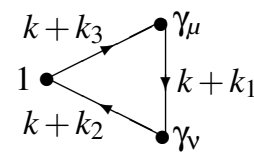

(b)

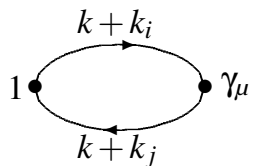

Figure 4: Diagrammatic representation for the $S V V$ three-point function and for the $S V$ two-point function, Figs.(a) and (b) respectively.

Contracting with the vector's vertexes momenta we can obtain a condition for the corresponding Ward identities

$$
\begin{aligned}
\left(k_{3}-k_{1}\right)^{\mu} T_{\mu v}^{S V V}= & \int \frac{d^{4} k}{(2 \pi)^{4}} \operatorname{Tr}\left\{\hat{1} \frac{1}{\left(k+k_{1}\right)-m} \gamma_{v} \frac{1}{\left(k+k_{2}\right)-m}\right\} \\
& -\int \frac{d^{4} k}{(2 \pi)^{4}} \operatorname{Tr}\left\{\hat{1} \frac{1}{\left(k+k_{3}\right)-m} \gamma_{v} \frac{1}{\left(k+k_{2}\right)-m}\right\},
\end{aligned}
$$

where we have used in the traces level the identity

and

$$
\left(k_{3}-k_{1}\right)=\left[k+k_{3}-m\right]-\left[k+k_{1}-m\right] .
$$

Let us now define the two-point function of the right hand side as (see Fig.4(b))

$$
T_{\mu \nu}^{S \rightarrow V V}=T_{\mu v}^{S V V}\left(k_{1}, k_{2}, k_{3} ; m\right)+T_{v \mu}^{S V V}\left(l_{1}, l_{2}, l_{3} ; m\right)
$$

$T_{\mu}^{V S}\left(k_{1}, k_{2} ; m\right)=\int \frac{d^{4} k}{(2 \pi)^{4}} \operatorname{Tr}\left\{\gamma_{\mu} \frac{1}{\left(k+k_{1}\right)-m} \hat{1} \frac{1}{\left(k+k_{2}\right)-m}\right\}$,

(34) in order to write the Ward identities as

$$
\begin{aligned}
p^{\mu} T_{\mu v}^{S \rightarrow V V} & =T_{v}^{V S}\left(k_{1}, k_{2} ; m\right)-T_{v}^{V S}\left(k_{3}, k_{2} ; m\right)+T_{v}^{V S}\left(l_{3}, l_{2} ; m\right)-T_{v}^{V S}\left(l_{3}, l_{1} ; m\right) \\
p^{\prime v} T_{\mu v}^{S \rightarrow V V} & =T_{\mu}^{V S}\left(k_{3}, k_{2} ; m\right)-T_{\mu}^{V S}\left(k_{3}, k_{1} ; m\right)+T_{v}^{V S}\left(l_{1}, l_{2} ; m\right)-T_{v}^{V S}\left(l_{3}, l_{2} ; m\right) .
\end{aligned}
$$

The conditions for the symmetry relations maintenance are put in terms of the value for the $S V$ two-point function structure. If the traces involved are performed we get

$$
\begin{aligned}
& T_{\mu}^{V S}=4 m\left\{\int \frac{d^{4} k}{(2 \pi)^{4}} \frac{2 k_{\mu}}{\left[\left(k+k_{1}\right)^{2}-m^{2}\right]\left[\left(k+k_{2}\right)^{2}-m^{2}\right]}\right. \\
&\left.+\left(k_{1}+k_{2}\right)_{\mu} \int \frac{d^{4} k}{(2 \pi)^{4}} \frac{1}{\left[\left(k+k_{1}\right)^{2}-m^{2}\right]\left[\left(k+k_{2}\right)^{2}-m^{2}\right]}\right\},
\end{aligned}
$$


which means that

$$
T_{\mu}^{V S}=4 m\left\{\left(k_{1}+k_{2}\right)_{\mu} I_{2}+2\left(I_{2}\right)_{\mu}\right\},
$$

or, given the definition (23),

$$
T_{\mu}^{V S}=4 m\left(\Delta I_{\mu}\right)
$$

If we look at the equation (22) of the preceding section we can see that the condition we have found for the Ward identities involved in the $S \rightarrow V V$ process is the same one we found for the complex scalar theory Ward identity. Only if the structure (40) is obtained identically vanishing, the symmetry relations are preserved by the one-loop perturbative calculation. Before the analysis let us now consider another (and more interesting) set of symmetry relations.

\section{IV. $A \rightarrow V V$ PROCESS}

A more interesting situation involving Ward identities emerges when we want to consider the process where an
Axial-Vector is connected with two vectors. Such a process can be constructed by coupling the appropriate fermionic densities with the external fields. Similar discussions can be found in the ref.[11] (see also ref.[10] and [12]). The interaction Lagrangian can be written as

$$
\mathcal{L}_{I}=i G_{P}\left(\bar{\Psi} \gamma_{5} \Psi\right) \pi-e_{V}\left(\bar{\Psi} \gamma^{\mu} \Psi\right) A_{\mu}-e_{A}\left(\bar{\Psi} \gamma_{5} \gamma^{\mu} \Psi\right) W_{\mu}^{A}
$$

Here, $\Psi$ is the massive $\frac{1}{2}$ fermion, $W_{\mu}^{A}$ is an Axial-Vector field and $\pi$ is a pseudo-scalar one. The fermionic currents obey

$$
\left\{\begin{array}{l}
\partial_{\mu} V^{\mu}=\partial_{\mu}\left(\bar{\Psi} \gamma^{\mu} \Psi\right)=0 \\
\partial_{\mu} A^{\mu}=\partial_{\mu}\left(\bar{\Psi} \gamma_{5} \gamma^{\mu} \Psi\right)=2 m i\left(\bar{\Psi} \gamma_{5} \Psi\right)=2 m i P .
\end{array}\right.
$$

In such theory we can define the Green's functions

$$
\begin{aligned}
& T_{\mu \vee \lambda}^{A \rightarrow V V}\left(p, p^{\prime} ; q\right)=i \int d^{4} x_{1} d^{4} x_{2} e^{i p x_{1}+i p^{\prime} x_{2}}<0\left|T\left(V_{\mu}\left(x_{1}\right) V_{\mu}\left(x_{2}\right) A_{\lambda}(0)\right)\right| 0> \\
& T_{\mu \nu}^{P \rightarrow V V}\left(p, p^{\prime} ; q\right)=i \int d^{4} x_{1} d^{4} x_{2} e^{i p x_{1}+i p^{\prime} x_{2}}<0\left|T\left(V_{\mu}\left(x_{1}\right) V_{v}\left(x_{2}\right) P_{0}(0)\right)\right| 0>.
\end{aligned}
$$

The standard procedure of current algebra can be used to state the Ward identities

$$
\begin{aligned}
p^{\prime \nu} T_{\lambda \mu \nu}^{A \rightarrow V V} & =0 \\
p^{\mu} T_{\lambda \mu \nu}^{A \rightarrow V V} & =0 \\
q^{\lambda} T_{\lambda \mu \nu}^{A \rightarrow V V} & =2 m T_{\mu \nu}^{P \rightarrow V V} .
\end{aligned}
$$

The lowest order perturbative calculation of the $A V V$ process requires the evaluation of the one-loop triangle diagrams of the Fig.5(a) and (b). The definitions for the external and internal lines follow the same conventions of the preceding section. So, we write for the direct channel (see Fig.5(a))

$$
T_{\lambda \mu v}^{A V V}\left(k_{1}, k_{2}, k_{3} ; m\right)=\int \frac{d^{4} k}{(2 \pi)^{4}} \operatorname{Tr}\left\{i \gamma_{\lambda} \gamma_{5} \frac{1}{(k+\not k)-m} \gamma_{\mu} \frac{1}{\left(\not k+k_{1}\right)-m} \gamma_{v} \frac{1}{\left(\not k+\not k_{2}\right)-m}\right\} .
$$

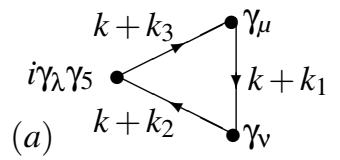

(a)

$k+k_{2} \cdot \gamma_{v}$

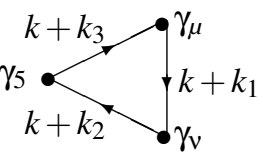

(c)

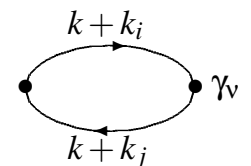

Figure 5: Diagrammatic representation for the $A V V$ and $P V V$ three-point functions and for the $A V$ two-point function, Figs.(a), (b) and (c), respectively.

Contracting with the external momenta we can derive conditions to be fulfilled in order to get the respective Ward identi- ties preserved. First we contract with the external momentum 
$\left(k_{3}-k_{2}\right)^{\lambda}$ and use the identity

in the interior of the traces, to get

$$
\left(k_{2}-k_{3}\right) \gamma_{5}=\left(\not k+k_{2}-m\right) \gamma_{5}+\gamma_{5}\left(k+k_{3}-m\right)+2 m \gamma_{5},
$$

$$
\begin{aligned}
\left(k_{3}-k_{2}\right)^{\lambda} T_{\lambda \mu \nu}^{A V V}= & -2 m i \int \frac{d^{4} k}{(2 \pi)^{4}} \operatorname{Tr}\left\{\gamma_{5} \frac{1}{[k+\not k 3-m]} \gamma_{\mu} \frac{1}{\left[k+\not k_{1}-m\right]} \gamma_{v} \frac{1}{[k+\not k-m]}\right\} \\
& -\int \frac{d^{4} k}{(2 \pi)^{4}} \operatorname{Tr}\left\{i \gamma_{v} \gamma_{5} \frac{1}{\left[k+\not k_{3}-m\right]} \gamma_{\mu} \frac{1}{\left[k+\not k_{1}-m\right]}\right\} \\
& +\int \frac{d^{4} k}{(2 \pi)^{4}} \operatorname{Tr}\left\{i \gamma_{\mu} \gamma_{5} \frac{1}{[k+\not k+m]} \gamma_{v} \frac{1}{[k+\not k 2-m]}\right\} .
\end{aligned}
$$

If we define the two-point functions on the right hand side as (see Fig. 5(c))

$$
T_{\xi \rho}^{A V}\left(k_{i}, k_{j} ; m\right)=\int \frac{d^{4} k}{(2 \pi)^{4}} \operatorname{Tr}\left\{i \gamma_{\xi} \gamma_{5} \frac{1}{\left(k+k_{i}\right)-m} \gamma_{\rho} \frac{1}{\left(k+k_{j}\right)-m}\right\},
$$

we can write (see Fig.6)

$$
\left(k_{3}-k_{2}\right)^{\lambda} T_{\lambda \mu \nu}^{A V V}=-2 i m T_{\mu \nu}^{P V V}-T_{v \mu}^{A V}\left(k_{3}, k_{1} ; m\right)+T_{\mu \nu}^{A V}\left(k_{1}, k_{2} ; m\right),
$$

where we have defined (see Fig.5(b))

$$
T_{\mu v}^{P V V}\left(k_{1}, k_{2}, k_{3} ; m\right)=\int \frac{d^{4} k}{(2 \pi)^{4}} \operatorname{Tr}\left\{\gamma_{5} \frac{1}{\left(k+\not k_{3}\right)-m} \gamma_{\mu} \frac{1}{\left(k+\not k_{1}\right)-m} \gamma_{v} \frac{1}{\left(\not k+\not k_{2}\right)-m}\right\} .
$$

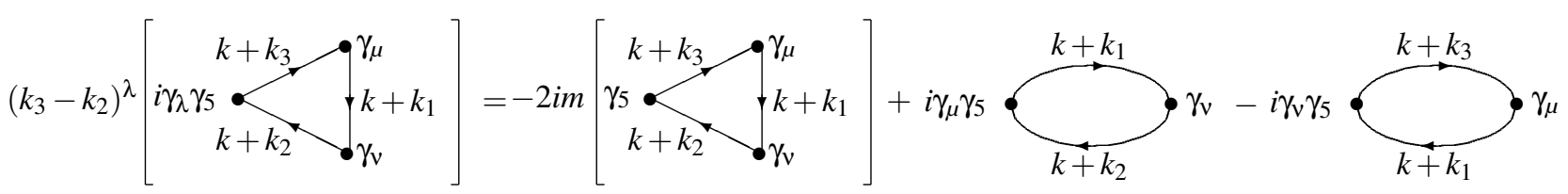

Figure 6: Diagrammatic representation for the identity (52).

Now if we take the contractions of the $A V V$ function with the vector's momenta we immediately identify (see Fig.7)

$$
\left(k_{3}-k_{1}\right)^{\mu} T_{\lambda \mu v}^{A V V}=T_{\lambda v}^{A V}\left(k_{1}, k_{2} ; m\right)-T_{\lambda v}^{A V}\left(k_{3}, k_{2} ; m\right) .
$$

Also, in a similar way we can state (see Fig.8)

$$
\left(k_{1}-k_{2}\right)^{v} T_{\lambda \mu \nu}^{A V V}=T_{\lambda \mu}^{A V}\left(k_{3}, k_{2} ; m\right)-T_{\lambda \mu}^{A V}\left(k_{3}, k_{1} ; m\right) .
$$

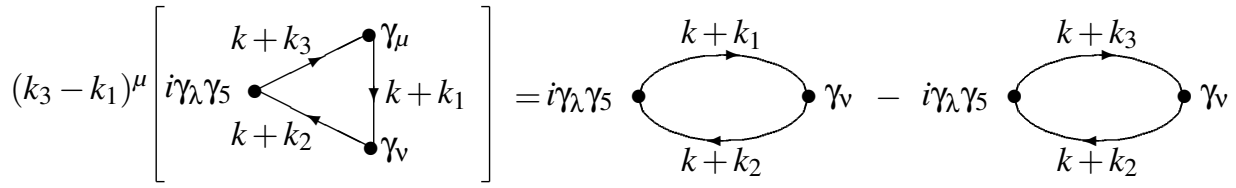

Figure 7: Diagrammatic representation for the identity (54).

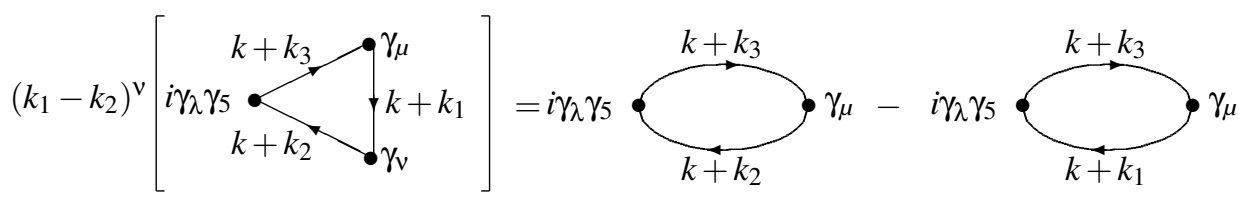

Figure 8: Diagrammatic representation for the identity (55). 
The inclusion of the crossed channel allows us to write the following expressions

$$
\begin{aligned}
q^{\lambda} T_{\lambda \mu \nu}^{A \rightarrow V V} & =-2 i m T_{\mu v}^{P \rightarrow V V}+T_{\mu v}^{A V}\left(k_{1}, k_{2} ; m\right)-T_{v \mu}^{A V}\left(k_{3}, k_{1} ; m\right)+T_{v \mu}^{A V}\left(l_{1}, l_{2} ; m\right)-T_{\mu \nu}^{A V}\left(l_{3}, l_{1} ; m\right), \\
p^{\mu} T_{\lambda \mu v}^{A \rightarrow V V} & =T_{\lambda \nu}^{A V}\left(k_{1}, k_{2} ; m\right)-T_{\lambda \nu}^{A V}\left(k_{3}, k_{2} ; m\right)+T_{\lambda \nu}^{A V}\left(l_{3}, l_{2} ; m\right)-T_{\lambda \nu}^{A V}\left(l_{3}, l_{1} ; m\right), \\
p^{\prime v} T_{\lambda \mu \nu}^{A \rightarrow V V} & =T_{\lambda \mu}^{A V}\left(k_{3}, k_{2} ; m\right)-T_{\lambda \mu}^{A V}\left(k_{3}, k_{1} ; m\right)+T_{\lambda \mu}^{A V}\left(l_{1}, l_{2} ; m\right)-T_{\lambda \mu}^{A V}\left(l_{3}, l_{2} ; m\right) .
\end{aligned}
$$

The conditions for Ward identities preservation are now put in terms of the $A V$ two-point functions. They are the same ones we can find in the ref.[10], [11] and [12]. The evaluation of the traces leads us to the expression

$$
\begin{aligned}
T_{\mu \nu}^{A V}\left(k_{1}, k_{2} ; m\right)=-4 & \varepsilon_{\mu v \alpha \beta}\left\{k_{2}^{\beta} \int \frac{d^{4} k}{(2 \pi)^{4}} \frac{k^{\alpha}}{\left[\left(k+k_{1}\right)^{2}-m^{2}\right]\left[\left(k+k_{2}\right)^{2}-m^{2}\right]}\right. \\
& +k_{1}^{\alpha} \int \frac{d^{4} k}{(2 \pi)^{4}} \frac{k^{\beta}}{\left[\left(k+k_{1}\right)^{2}-m^{2}\right]\left[\left(k+k_{2}\right)^{2}-m^{2}\right]} \\
& \left.+k_{1}^{\alpha} k_{2}^{\beta} \int \frac{d^{4} k}{(2 \pi)^{4}} \frac{1}{\left[\left(k+k_{1}\right)^{2}-m^{2}\right]\left[\left(k+k_{2}\right)^{2}-m^{2}\right]}\right\} .
\end{aligned}
$$

We can use the properties of the totally antisymmetric tensor $\varepsilon_{\mu v \alpha \beta}$ in order to put the equation above into the form

$$
T_{\mu \nu}^{A V}\left(k_{1}, k_{2} ; m\right)=2 \varepsilon_{\mu v \alpha \beta}\left(k_{1}-k_{2}\right)^{\beta}\left\{\left(k_{1}+k_{2}\right)^{\alpha} I_{2}+2\left(I_{2}\right)^{\alpha}\right\} .
$$

This means that, given the definition (23), we have

$$
T_{\mu v}^{A V}\left(k_{1}, k_{2} ; m\right)=2 \varepsilon_{\mu v \alpha \beta}\left(k_{1}-k_{2}\right)^{\beta}\left(\Delta I^{\alpha}\right),
$$

which means that the condition is the same one as those found in the preceding sections. Now it is time to study the divergent integrals that appeared in the three amplitudes considered and their symmetry relations.

\section{THE CALCULATIONAL STRATEGY}

If the explicit evaluation of perturbative (divergent) amplitudes is in order we need to specify some philosophy to handle the mathematical indefinitions involved. Usually the calculations become reliable only after the adoption of a regularization technique. After this, in the intermediary steps, we invariably assume some specific consequences for the results intrinsically associated to the properties attributed for the divergent integrals resulting from the (arbitrary) choices for the mathematical indefinitions implied by the adopted regularization. In the final form this way obtained, for the amplitudes in general, it is not possible to specify in a clear way what are the particular effects of the adopted regularization for the result or, in other words, to evaluate in what sense the expression is dependent on the adopted technique. In order to perform a as safe as possible analysis of the properties of the divergent amplitudes, including their symmetry relations and the question of the ambiguities related to the arbitrariness involved in the routing of the loop internal lines momenta, we need to avoid as much as possible specific choices in the intermediary steps so that all the possibilities still remain contained in the final results. If it is possible, we can change the usual focus of the analysis, which is the verification by testing the consistency of the proposed regularization technique, for the identification of the eventual properties such a technique should have in order to be consistent. The implication of the preceding arguments, which will become clear in what follows, will play an important role in the discussion we want to perform.

To explicitly evaluate the divergent integrals involved we will adopt an alternative strategy to handle the divergences. The referred method, introduced in ref. [7], has been used recently in the literature in different contexts. It allows us a clear and universal point of view for the divergences of perturbative calculations in QFT. The strategy is simple: instead of the specification of some regularization, to justify all the necessary manipulations, we will assume the presence of a regulating distribution only in an implicit way. Schematically

$$
\begin{aligned}
\int \frac{d^{4} k}{(2 \pi)^{4}} f(k) & \rightarrow \int \frac{d^{4} k}{(2 \pi)^{4}} f(k)\left\{\lim _{\Lambda_{i}^{2} \rightarrow \infty} G_{\Lambda_{i}}\left(k, \Lambda_{i}^{2}\right)\right\} \\
& =\int_{\Lambda} \frac{d^{4} k}{(2 \pi)^{4}} f(k)
\end{aligned}
$$

Here $\Lambda_{i}^{\prime} s$ are parameters of the generic distribution $G\left(\Lambda_{i}^{2}, k\right)$ that, in addition to the obvious finite character of the modified integral, must have two other very general properties. It must be even in the integrating momentum $k$, due to Lorentz invariance maintenance, as well as a well-defined connection limit must exists, i.e.,

$$
\lim _{\Lambda_{i}^{2} \rightarrow \infty} G_{\Lambda_{i}}\left(k^{2}, \Lambda_{i}^{2}\right)=1
$$

The first property imply that all odd integrals vanish. The second one guarantees, in particular, that the value of the finite integrals in the amplitudes will not be modified. Having this in mind, we manipulate the integrand of the divergent integrals to generate a mathematical expression where all the divergences are located in internal momenta independent structures. This goal can be achieved by the use of an adequate 
identity like

$$
\begin{aligned}
\frac{1}{\left[\left(k+k_{i}\right)^{2}-m^{2}\right]} & =\sum_{j=0}^{N} \frac{(-1)^{j}\left(k_{i}^{2}+2 k_{i} \cdot k\right)^{j}}{\left(k^{2}-m^{2}\right)^{j+1}} \\
& +\frac{(-1)^{N+1}\left(k_{i}^{2}+2 k_{i} \cdot k\right)^{N+1}}{\left(k^{2}-m^{2}\right)^{N+1}\left[\left(k+k_{i}\right)^{2}-m^{2}\right]}
\end{aligned}
$$

where $k_{i}$ is (in principle) an arbitrary choice for the routing of a loop internal line momentum. The value for $\mathrm{N}$ must be adequately chosen. The minor value must be the one that leads the last term in the above expression to be present in a finite integral and therefore, by virtue of the well-defined connection limit assumptions, the corresponding integration can be performed without any restrictions and free from the specific effects of the eventual regularization. All the remaining structures become independent on the internal lines momenta. We then eliminate all the integrals with odd integrand, as a trivial consequence of the even character of the regulating implicit distribution. In the divergent structures obtained this way no additional assumptions are taken. They are organized in five objects, namely

$$
\begin{aligned}
\square_{\alpha \beta \mu \nu}= & \int_{\Lambda} \frac{d^{4} k}{(2 \pi)^{4}} \frac{24 k_{\mu} k_{v} k_{\alpha} k_{\beta}}{\left(k^{2}-m^{2}\right)^{4}}-g_{\alpha \beta} \int_{\Lambda} \frac{d^{4} k}{(2 \pi)^{4}} \frac{4 k_{\mu} k_{\nu}}{\left(k^{2}-m^{2}\right)^{3}} \\
& -g_{\alpha \nu} \int_{\Lambda} \frac{d^{4} k}{(2 \pi)^{4}} \frac{4 k_{\beta} k_{\mu}}{\left(k^{2}-m^{2}\right)^{3}}-g_{\alpha \mu} \int_{\Lambda} \frac{d^{4} k}{(2 \pi)^{4}} \frac{4 k_{\beta} k_{v}}{\left(k^{2}-m^{2}\right)^{3}}, \\
\Delta_{\mu v}= & \int_{\Lambda} \frac{d^{4} k}{(2 \pi)^{4}} \frac{4 k_{\mu} k_{v}}{\left(k^{2}-m^{2}\right)^{3}}-\int_{\Lambda} \frac{d^{4} k}{(2 \pi)^{4}} \frac{g_{\mu \nu}}{\left(k^{2}-m^{2}\right)^{2}}, \\
\nabla_{\mu v}= & \int_{\Lambda} \frac{d^{4} k}{(2 \pi)^{4}} \frac{2 k_{v} k_{\mu}}{\left(k^{2}-m^{2}\right)^{2}}-\int_{\Lambda} \frac{d^{4} k}{(2 \pi)^{4}} \frac{g_{\mu \nu}}{\left(k^{2}-m^{2}\right)}, \\
I_{\text {log }}\left(m^{2}\right)= & \int_{\Lambda} \frac{d^{4} k}{(2 \pi)^{4}} \frac{1}{\left(k^{2}-m^{2}\right)^{2}}, \\
I_{\text {quad }}\left(m^{2}\right)= & \int_{\Lambda} \frac{d^{4} k}{(2 \pi)^{4}} \frac{1}{\left(k^{2}-m^{2}\right)} .
\end{aligned}
$$

This systematization is sufficient for discussions in fundamental theories at the one-loop level. In the non-renormalizable ones new objects can be defined following this philosophy. In the two (or more) loop level of calculations new basic divergent structures can be equally defined in a completely analogous way. The main point is to avoid the explicit evaluation of such divergent structures in which case a regulating distribution needs to be specified.

We can say that this procedure furnishes an universal point of view for the calculated amplitudes since it become possible to map the final expressions obtained this way into the corresponding results of other techniques. All the steps followed and all the assumptions are perfectly valid in the reasonable regularization prescriptions, including the DR. All we need, to extract from our results those of a specific technique, is to evaluate the divergent structures, remaining at the final expression, according to the specific chosen prescription. Another important fact we call the attention is that no shifts or expansions are used in the intermediary steps. We assume the more general routing for all amplitudes. The potentially ambiguous terms are still present in the final result. Consequently, it is possible to make contact with those results corresponding to explicit evaluation of surface's terms involved when shifts in the integrating momenta are performed. This is an important aspect of our analysis because we want to make contact with the traditional approach used to justify the triangle anomalies.

In order to clarify the above described method, to handle the divergences, let us apply the calculational strategy in the treatment for some divergent integrals. For this purposes we take two of them that will play an important role in our analysis. They are two-point function structures defined in eq.(24). The $I_{2}$ integral, is a logarithmically divergent structure while $\left(I_{2}\right)_{\mu}$ is linearly divergent. In this structures $k_{1}$ and $k_{2}$ represents, in principle, arbitrary choices for the internal lines momenta. Then we can expect a dependence on $k_{1}$ and $k_{2}$ other than the difference between them only for the $\left(I_{2}\right)_{\mu}$ integral.

Taken first the $I_{2}$ integral we choose, in the identity (64), $N=1$ to rewrite both denominators. Then we get

$$
\begin{aligned}
I_{2}= & \int_{\Lambda} \frac{d^{4} k}{(2 \pi)^{4}} \frac{1}{\left(k^{2}-m^{2}\right)^{2}} \\
& -\int \frac{d^{4} k}{(2 \pi)^{4}} \frac{\left(k_{1}^{2}+2 k_{1} \cdot k\right)^{2}}{\left[\left(k^{2}-m^{2}\right)^{2}\right]\left[\left(k+k_{1}\right)^{2}-m^{2}\right]} \\
& -\int \frac{d^{4} k}{(2 \pi)^{4}} \frac{\left(k_{2}^{2}+2 k_{2} \cdot k\right)^{2}}{\left[\left(k^{2}-m^{2}\right)^{2}\right]\left[\left(k+k_{2}\right)^{2}-m^{2}\right]} \\
& +\int \frac{d^{4} k}{(2 \pi)^{4}} \frac{\left(k_{1}^{2}+2 k_{1} \cdot k\right)\left(k_{2}^{2}+2 k_{2} \cdot k\right)}{\left[\left(k^{2}-m^{2}\right)^{2}\right]\left[\left(k+k_{1}\right)^{2}-m^{2}\right]\left[\left(k+k_{2}\right)^{2}-m^{2}\right]} .
\end{aligned}
$$


The right hand side exhibits the desirable form. The divergent term is located in an internal momenta independent structure which we can identify as $I_{\log }\left(m^{2}\right)$, defined in eq.(68). The remaining structures are finite ones and we use what we call the connection limit existence to drop the $\Lambda$ subscript on the integral, or, equivalently, to remove the eventual regulating distribution under the argumentation that the integration and the connection limit can be perfectly interchanged. The thus obtained finite Feynman integrals can be solved without any problem. The answer can be written as

$$
I_{2}=I_{l o g}\left(m^{2}\right)-\left(\frac{i}{(4 \pi)^{2}}\right) Z_{0}\left(\left(k_{1}-k_{2}\right)^{2} ; m^{2}\right),
$$

where we have introduced (in short hand notation) the twopoint functions structures [7]

$Z_{k}\left(\lambda_{1}^{2}, \lambda_{2}^{2}, q^{2} ; \lambda^{2}\right)=\int_{0}^{1} d z z^{k} \ln \left(\frac{q^{2} z(1-z)+\left(\lambda_{1}^{2}-\lambda_{2}^{2}\right) z-\lambda_{1}^{2}}{\left(-\lambda^{2}\right)}\right)$

Integration over parameter $z$ can be easily performed however for our present purposes this is not necessary.

Following the procedure we can evaluate also the $I_{2}^{\mu}$ integral. The first step is the same: the use of the identity (64) to rewrite the integrand, now to the form

$$
\begin{aligned}
& \left(I_{2}\right)_{\mu}=-\frac{1}{2}\left(k_{1}+k_{2}\right)^{\alpha} \int_{\Lambda} \frac{d^{4} k}{(2 \pi)^{4}} \frac{4 k_{\alpha} k_{\mu}}{\left(k^{2}-m^{2}\right)^{3}} \\
& +\int \frac{d^{4} k}{(2 \pi)^{4}} \frac{\left(k_{1}^{2}+2 k_{1} \cdot k\right)^{2} k_{\mu}}{\left[\left(k^{2}-m^{2}\right)^{3}\right]\left[\left(k+k_{1}\right)^{2}-m^{2}\right]} \\
& +\int \frac{d^{4} k}{(2 \pi)^{4}} \frac{\left(k_{2}^{2}+2 k_{2} \cdot k\right)^{2} k_{\mu}}{\left[\left(k^{2}-m^{2}\right)^{3}\right]\left[\left(k+k_{2}\right)^{2}-m^{2}\right]} \\
& +\int \frac{d^{4} k}{(2 \pi)^{4}} \frac{\left(k_{1}^{2}+2 k_{1} \cdot k\right)\left(k_{2}^{2}+2 k_{2} \cdot k\right) k_{\mu}}{\left[\left(k^{2}-m^{2}\right)^{2}\right]\left[\left(k+k_{1}\right)^{2}-m^{2}\right]\left[\left(k+k_{2}\right)^{2}-m^{2}\right]} .
\end{aligned}
$$

In the above expression, we have dropped two odd $k$ integrals, by virtue of the even character of the implicit regulating distribution as well as the $\Lambda$ subscript in the last three terms due to the finite character. After the integration of the finite terms we are lead to the result

$$
\begin{aligned}
\left(I_{2}\right)_{\mu} & =-\frac{1}{2}\left(k_{1}+k_{2}\right)^{\alpha}\left(\Delta_{\alpha \mu}\right)-\frac{1}{2}\left(k_{1}+k_{2}\right)_{\mu}\left\{I_{\log }\left(m^{2}\right)\right. \\
& \left.-\left(\frac{i}{(4 \pi)^{2}}\right) Z_{0}\left(\left(k_{1}-k_{2}\right)^{2} ; m^{2}\right)\right\} \\
& =-\frac{1}{2}\left(k_{1}+k_{2}\right)^{\alpha}\left(\Delta_{\alpha \mu}\right)-\frac{1}{2}\left(k_{1}+k_{2}\right)_{\mu}\left(I_{2}\right) .
\end{aligned}
$$

It is important, at this point, to emphasize the general aspects of the method. No shifts has been performed and, in fact, no divergent integrals calculated. All the final results produced by this approach can be mapped in those of any specific technique. The finite parts are the same as should be by physical reasons. The divergent parts can be easily obtained. All we need is to evaluate the remaining divergent structures according to the chosen prescription. By virtue of this general character, the present strategy can be used simply to systematize the procedures, even if one wants to use traditional techniques. Let us now to use the above obtained results to calculate the physical amplitudes involved in our present discussions.

\section{DIVERGENCES, AMBIGUITIES AND WARD IDENTITIES}

Given the results obtained in the preceding section we can evaluate the combination of Feynman integrals which revealed being crucial for all the Ward identities we have studied. Substituting the results (71) and (74) in the expression (23) we get

$$
\Delta I_{\mu}=\left(k_{1}+k_{2}\right)^{\alpha}\left(\triangle_{\alpha \mu}\right),
$$

and consequently,

$$
\begin{aligned}
& T_{\mu}^{V S}\left(k_{1}, k_{2} ; m\right)=-4 m\left(k_{1}+k_{2}\right)^{\alpha}\left(\triangle_{\alpha \mu}\right) \\
& T_{\mu \nu}^{A V}\left(k_{1}, k_{2} ; m\right)=2 \varepsilon_{\mu v \alpha \beta}\left(k_{1}-k_{2}\right)^{\alpha}\left\{\left(k_{1}+k_{2}\right)^{\xi}\left(\triangle_{\xi}^{\beta}\right)\right\}
\end{aligned}
$$

The Ward identities we studied in the sections II, III and IV can be written into the following form

$$
\begin{aligned}
i q^{\mu} \Gamma_{\mu}^{1 \text { loop }}(p, q)= & i \lambda\left(k_{1}-k_{2}\right)^{\mu}\left(k_{1}+k_{2}\right)^{\alpha}\left(\triangle_{\alpha \mu}\right), \\
q^{\lambda} T_{\lambda \mu \nu}^{A \rightarrow V V}= & -2 m i\left[T_{\mu v}^{P \rightarrow V V}\right]+2 \varepsilon_{\mu v \alpha \beta}\left[\left(k_{1}-k_{3}\right)^{\beta}\left(k_{1}+k_{3}\right)^{\xi}+\left(k_{2}-k_{1}\right)^{\beta}\left(k_{1}+k_{2}\right)^{\xi}\right]\left(\triangle_{\xi}^{\alpha}\right) \\
& -2 \varepsilon_{\mu v \alpha \beta}\left[\left(l_{1}-l_{3}\right)^{\beta}\left(l_{1}+l_{3}\right)^{\xi}+\left(l_{2}-l_{1}\right)^{\beta}\left(l_{1}+l_{2}\right)^{\xi}\right]\left(\triangle_{\xi}^{\alpha}\right), \\
p^{\mu} T_{\lambda \mu \nu}^{A \rightarrow V V}= & 2 \varepsilon_{\lambda v \alpha \beta}\left[\left(k_{2}-k_{1}\right)^{\beta}\left(k_{1}+k_{2}\right)^{\xi}+\left(k_{3}-k_{2}\right)^{\beta}\left(k_{2}+k_{3}\right)^{\xi}\right]\left(\triangle_{\xi}^{\alpha}\right) \\
& +2 \varepsilon_{\lambda v \alpha \beta}\left[\left(l_{3}-l_{1}\right)^{\beta}\left(l_{1}+l_{3}\right)^{\xi}+\left(l_{2}-l_{3}\right)^{\beta}\left(l_{2}+l_{3}\right)^{\xi}\right]\left(\triangle_{\xi}^{\alpha}\right),
\end{aligned}
$$




$$
\begin{aligned}
p^{\prime v} T_{\lambda \mu \nu}^{A \rightarrow V V}= & 2 \varepsilon_{\lambda \mu \alpha \beta}\left[\left(k_{3}-k_{1}\right)^{\beta}\left(k_{1}+k_{3}\right)^{\xi}+\left(k_{2}-k_{3}\right)^{\beta}\left(k_{2}+k_{3}\right)^{\xi}\right]\left(\triangle_{\xi}^{\alpha}\right) \\
& +2 \varepsilon_{\lambda \mu \alpha \beta}\left[\left(l_{2}-l_{1}\right)^{\beta}\left(l_{1}+l_{2}\right)^{\xi}+\left(l_{3}-l_{2}\right)^{\beta}\left(l_{2}+l_{3}\right)^{\xi}\right]\left(\triangle_{\xi}^{\alpha}\right), \\
p^{\mu} T_{\mu \nu}^{S \rightarrow V V}= & 4 m\left(k_{3}-k_{1}\right)^{\xi}\left(\triangle_{\xi v}\right)+4 m\left(l_{1}-l_{2}\right)^{\xi}\left(\triangle_{\xi v}\right)=8 m p^{\xi}\left(\triangle_{\xi v}\right), \\
p^{\prime \nu} T_{\lambda \mu \nu}^{S \rightarrow V V}= & 4 m\left(k_{1}-k_{2}\right)^{\xi}\left(\triangle_{\xi \mu}\right)+4 m\left(l_{3}-l_{1}\right)^{\xi}\left(\triangle_{\xi \mu}\right)=8 m p^{\prime \xi}\left(\triangle_{\xi \mu}\right) .
\end{aligned}
$$

There are two types of undefined quantities in the expressions above. This means that in order to get definite results for the involved amplitudes it becomes necessary to assume some (arbitrary) choices for them. Such choices must be obviously guided by the consistency we want to get in perturbative calculations, in spite of the divergent character. Having this in mind we can ask for the existence of eventual physical constraints to be used in order to get the adequate choices for the arbitrariness present in the results above. Clearly, there are two types of constraints which we must fulfill. The first one refers to the Ward identities themselves, i.e., we want to make choices that lead, in principle, to their preservation. For the second, we cannot forget that the conditions (78)-(83) are obtained after the evaluation of the $A V$ and $S V$ two-point functions, so that our choices for the arbitrariness must not imply in non-physical results for these amplitudes. In addition, we note that these two amplitudes are deeply related. There is an identity at the traces level relating them, namely (see Figure 9):

$$
\begin{aligned}
& T_{\mu \nu}^{A V}=-\frac{1}{2 m} \varepsilon_{\mu \nu \alpha \beta}\left(k_{1}-k_{2}\right)^{\alpha}\left(T^{V S}\right)^{\beta} . \\
& i \gamma_{\mu} \gamma_{5} \overbrace{k+k_{j}}^{k+k_{i}} \gamma_{v}=\frac{1}{2 m} \varepsilon_{\mu v \alpha \beta}\left(k_{j}-k_{i}\right)^{\alpha} \overbrace{k+k_{j}}^{k+k_{i}} \gamma^{\beta}]
\end{aligned}
$$

Figure 9: Diagrammatic representation for the identity (84).

The identity is valid before any calculations have been made, i.e., independent of the divergences related aspect involved. It must be valid after all the calculations are performed independent of the adopted regularization philosophy. Within our calculational strategy, the above identity is preserved before any choices for the arbitrariness involved. Both amplitudes are, in principle, ambiguous quantities. The identity (84) is not ambiguous and should be maintained after the adopted interpretations for the arbitrariness involved. Given this observation it is natural to start by the analysis of the $S V$ and $A V$ two-point functions. First, by unitarity reasons (Cutkosky's rules), both two-point functions should have an imaginary part arising at the kinematical point $\left(k_{1}-k_{2}\right)^{2}=$ $4 m^{2}$. If a nonzero value for the referred amplitudes is attributed, independent of the possible choices involved, such a threshold will not be present. For the second, Lorentz and $\mathrm{CPT}$ arguments also require the vanishing value. Otherwise, a transition between a vector into a scalar particle and between an axial into a vector particle seems to be possible through the $S V$ and $A V$ two-point functions respectively. We can also add some arguments coming from Ward identities. The $S V$ amplitude carries a vector Lorentz index in such a way the contraction with the external momentum of the re- spective vertex should vanish, in order to maintain the vector current conserved. Given the expression (76), the contraction with $\left(k_{1}-k_{2}\right)^{\mu}$ gives us

$$
\left(k_{1}-k_{2}\right)^{\mu} T_{\mu}^{V S}=-4 m\left(k_{1}-k_{2}\right)^{\mu}\left(k_{1}+k_{2}\right)^{\xi}\left[\triangle_{\xi \mu}\right],
$$

which is not automatically zero. On the other hand, the $A V$ amplitude should also exhibit a conserved vector current. The contraction of the expression (77) with $\left(k_{1}-k_{2}\right)^{\mu}$, due to the symmetry properties of the $\varepsilon_{\mu v \alpha \beta}$ tensor, gives us

$$
\left(k_{1}-k_{2}\right)^{\mu} T_{\mu \nu}^{A V}=0 \text {. }
$$

This is therefore a good property. However, by the same reasons put above, we get

$$
\left(k_{1}-k_{2}\right)^{v} T_{\mu \nu}^{A V}=0,
$$

which means that the axial current is also conserved, if a nonzero value for the $A V$ structure is assumed. This is bad because the correct result is a proportionality between the axial and pseudo-scalar current, as it is well-known. Only the zero value for both mathematical structures allows the correct symmetry content of both considered two-point functions. 
Given this conclusion the question immediately raised is: how can we use the arbitrariness remaining in the expressions (76) and (77) in order to obtain the desirable results? Looking at the structure of the results (76) and (77) we see that there are two basic possibilities. First, due to the fact that the value for $k_{1}+k_{2}$ is not fixed by the energy-momentum conservation, we can choose $k_{1}=q / 2$ and $k_{2}=-q / 2$ where $q$ is the external momentum. Within this procedure the value for the object $\Delta_{\mu v}$ is not constrained and all regularizations can be used to evaluate it. Secondly, since we need to calculate the value for $\Delta_{\mu \nu}$, i.e., to adopt a regularization, we can select it in such a way that $\Delta_{\mu \nu}^{r e g}=0$. Both choices should impose a price to be paid in other calculations if we want to construct a procedure, i.e., if we assume that all the amplitudes in all theories and models must be treated in the same way. The first possibility pointed above implies in the assumption that the amplitudes may emerge ambiguous from the calculations, i.e., dependent on the choices for the internal lines labels. This is bad because the predictive power of QFT in the perturbative approach is destroyed and, as a consequence, we can use the theory only to produce adjustments to well-known phenomenologies. The predictions cannot be stated in general because the amplitudes may have undefined pieces. In addition, in adopting this way, we are assuming that the space-time homogeneity is broken in the calculations. If, on the other hand, we take the second option there are also some implications. Specific properties for the divergent integrals are assumed and they need to be used in all other calculations with the same value and exhibiting the same consistency, which, in fact, should be verified.

After these important remarks we return to the Ward identities (78)-(83). Looking at the Ward identities for the complex scalar field we note that there are two types of arbitrariness involved; the presence of the undefined piece $\Delta$ and the ambiguous combination of the external lines momenta $k_{1}+k_{2}$. We have both options described above in order to get a symmetry preserving result. A different situation occurs in the $S V V$ symmetry relation. Even that the condition for the symmetry preservation was put in terms of four potentially ambiguous terms they appear in non-ambiguous combinations. So all the choices for the internal arbitrary momenta lead us to the same result. Only the choice $\Delta_{\mu \nu}^{r e g}=0$ will give us the desirable result. We note that these two problems, the scalar field and $S V V$ process, can be treated within the DR. In fact, the strategy we have used to perform the calculations, with the choice $\Delta_{\mu v}^{r e g}=0$, becomes identical to the DR in theories with only bosonic fields and mappable one-by-one in theories with fermions. The $S V$ amplitude is trivially obtained identically zero in the DR. Then, it seems obvious that all the physical requirements are fulfilled by the choice $\Delta_{\mu v}^{r e g}=0$, which maps the DR results. What are the reasons for hesitation in assuming this option? The answer can be extracted from the consequences of this choice for the Ward identities (78)-(83): they become

$$
\begin{aligned}
i q^{\mu} \Gamma_{\mu}^{1 \text { loop }}(p, q) & =0, \\
p^{\prime \nu} T_{\mu v}^{S \rightarrow V V} & =0, \\
p^{\mu} T_{\mu v}^{S \rightarrow V V} & =0,
\end{aligned}
$$

and

$$
\begin{aligned}
& p^{\prime \nu} T_{\lambda \mu \nu}^{A \rightarrow V V}=0, \\
& p^{\mu} T_{\lambda \mu \nu}^{A \rightarrow V V}=0, \\
& q^{\lambda} T_{\lambda \mu \nu}^{A \rightarrow V V}=-2 m i T_{\mu \nu}^{P \rightarrow V V},
\end{aligned}
$$

i.e., all the Ward identities become preserved and all the ambiguities are automatically eliminated. At first sight this fact can be understood as a trouble because, apparently, we are forbidding any violation of symmetry relations in the $A V V$ triangle amplitude which is well known should present an anomaly to be consistent with the electromagnetic pion decay. At least this is the line of reasoning which we can find in the traditional literature about this issue. In order to justify the anomaly it is assumed that the undefined terms on the right hand side of the eqs.(79)-(81), which are in the last instance $A V$ physical amplitudes, are non-vanishing and ambiguous. In order to make clear the last sentence let us consider the recovering of the results corresponding to what we call the traditional treatment from the ones of the adopted calculational strategy. The referred results can be easily found in the literature about the subject or in many textbooks of QFT. It is a very simple job to pass from our results to the ones corresponding to the surface's terms evaluation due to the fact that no shifts have been made in the intermediary steps. All we need is: first to state the identities (55)-(57), and then to evaluate the twopoint function structures thus obtained, with the help of the results (71) and (74), which lead us to the expression

$$
\begin{aligned}
&\left(k_{3}-k_{2}\right)^{\lambda} T_{\lambda \mu \nu}^{A V V}=-2 m i\left[T_{\mu \nu}^{P V V}\right]+2 \varepsilon_{\mu v \alpha \beta}\left[\left(k_{1}-k_{3}\right)^{\beta}\left(k_{1}+k_{3}\right)^{\xi}+\left(k_{2}-k_{1}\right)^{\beta}\left(k_{1}+k_{2}\right)^{\xi}\right]\left(\triangle_{\xi}^{\alpha}\right), \\
&\left(k_{3}-k_{1}\right)^{\mu} T_{\lambda \mu \nu}^{A V V}=2 \varepsilon_{\lambda \nu \alpha \beta}\left[\left(k_{2}-k_{1}\right)^{\beta}\left(k_{1}+k_{2}\right)^{\xi}+\left(k_{3}-k_{2}\right)^{\beta}\left(k_{2}+k_{3}\right)^{\xi}\right]\left(\triangle_{\xi}^{\alpha}\right), \\
&\left(k_{1}-k_{2}\right)^{v} T_{\lambda \mu v}^{A V V}=2 \varepsilon_{\lambda \mu \alpha \beta}\left[\left(k_{3}-k_{1}\right)^{\beta}\left(k_{1}+k_{3}\right)^{\xi}+\left(k_{2}-k_{3}\right)^{\beta}\left(k_{2}+k_{3}\right)^{\xi}\right]\left(\triangle_{\xi}^{\alpha}\right) .
\end{aligned}
$$

Now we observe that, in order to give a definite value for the right hand side of the equations, two types of arbitrariness need to be removed by taking choices. Such arbitrariness are the ambiguous combinations of internal momenta and the un- 
defined mathematical object. The difference between two logarithmically divergent integrals, however, can be immediately identified with a typical surface's term and easily evaluated as follows

$$
\begin{aligned}
\Delta S_{\mu v} & =\int_{\Lambda} \frac{d^{4} k}{(2 \pi)^{4}} \frac{\partial}{\partial k_{v}}\left(\frac{k_{\mu}}{\left(k^{2}-m^{2}\right)^{2}}\right) \\
& =\int_{\Lambda} \frac{d^{4} k}{(2 \pi)^{4}} \frac{-4 k_{\mu} k_{v}}{\left(k^{2}-m^{2}\right)^{3}}+\int_{\Lambda} \frac{d^{4} k}{(2 \pi)^{4}} \frac{g_{\mu v}}{\left(k^{2}-m^{2}\right)^{2}} \\
& =\left(\frac{i}{(4 \pi)^{2}}\right)\left(\frac{1}{2}\right) g_{\mu v} .
\end{aligned}
$$

Note that the same result could be obtained by shifting the integrating momentum in one of the two-point function structures in order to produce a cancellation with the other one. The price to be paid, which is well known, is the addition of the corresponding surface's term which assumes exactly the value obtained above. The next step is the removal of the undefined combination of internal momenta. We adopt then a parametrization for the internal momenta in terms of the external ones

$$
\left\{\begin{array}{l}
k_{1}=a p^{\prime}+b p \\
k_{2}=b p+(a-1) p^{\prime} \\
k_{3}=a p^{\prime}+(b+1) p .
\end{array}\right.
$$

where $a$ and $b$ are constants. Notice that : $k_{1}-k_{2}=p^{\prime}, k_{3}-$ $k_{1}=p$ and $k_{3}-k_{2}=p^{\prime}+p=q$, where $q$ is obviously the momentum of the axial vector. After these substitutions we get

$$
\begin{aligned}
\left(k_{3}-k_{2}\right)^{\lambda} T_{\lambda \mu \nu}^{A V V} & =-2 m i T_{\mu \nu}^{P V V}-\frac{(a-b)}{8 \pi^{2}} i \varepsilon_{\mu v \xi \eta} p^{\prime \eta} p^{\xi} \\
\left(k_{3}-k_{1}\right)^{\mu} T_{\lambda \mu \nu}^{A V V} & =-\frac{(1-a)}{8 \pi^{2}} i \varepsilon_{\lambda v \xi \eta} p^{\prime \eta} p^{\xi} \\
\left(k_{1}-k_{2}\right)^{v} T_{\lambda \mu \nu}^{A V V} & =\frac{(1+b)}{8 \pi^{2}} i \varepsilon_{\lambda \mu \xi \eta} p^{\prime \eta} p^{\xi}
\end{aligned}
$$

In the expressions above it remains the arbitrariness related to the routing of internal lines now present in the parameters $a$ and $b$. In addition we note that there are no values for $a$ and $b$ in such a way that all the expected relations among the involved Green's functions are simultaneously satisfied. If we follow this line of reasoning and include the contribution of the crossed diagram whose parametrization for the internal lines momenta can be assumed as

$$
\left\{\begin{array}{l}
l_{1}=c p+d p^{\prime} \\
l_{2}=d p^{\prime}+(c-1) p \\
l_{3}=c p+(d+1) p^{\prime},
\end{array}\right.
$$

we will obtain

$$
\begin{aligned}
\left(l_{3}-l_{2}\right)^{\lambda} T_{\lambda v \mu}^{A V V} & =-2 m i T_{v \mu}^{P V V}-\frac{(c-d)}{8 \pi^{2}} i \varepsilon_{\mu v \xi \eta} p^{\prime \eta} p^{\xi} \\
\left(l_{1}-l_{2}\right)^{\mu} T_{\lambda v \mu}^{A V V} & =-\frac{(d+1)}{8 \pi^{2}} i \varepsilon_{\lambda v \xi \eta} p^{\prime \eta} p^{\xi} \\
\left(l_{3}-l_{1}\right)^{v} T_{\lambda v \mu}^{A V V} & =-\frac{(c-1)}{8 \pi^{2}} i \varepsilon_{\lambda \mu \xi \eta} p^{\prime \eta} p^{\xi}
\end{aligned}
$$

The addition of the two contributions gives us

$$
\begin{aligned}
q^{\lambda} T_{\lambda \mu \nu}^{A \rightarrow V V} & =-2 m i T_{\mu \nu}^{P \rightarrow V V}-\frac{(a-b+c-d)}{8 \pi^{2}} i \varepsilon_{\mu v \xi \eta} p^{\prime \eta}\left(1 \xi^{\xi}, 5\right) \\
p^{\mu} T_{\lambda \mu \nu}^{A \rightarrow V V} & =-\frac{(d-a+2)}{8 \pi^{2}} i \varepsilon_{\lambda v \xi \eta} p^{\prime \eta} p^{\xi}, \\
p^{\prime \nu} T_{\lambda \mu \nu}^{A \rightarrow V V} & =-\frac{(c-b-2)}{8 \pi^{2}} i \varepsilon_{\lambda \mu \xi \eta} p^{\prime \eta} p^{\xi} .
\end{aligned}
$$

A closer contact with the usual results can be obtained if it is assumed the same significance for the arbitrary internal momenta, i.e., $a=c$ and $b=d$ in the eqs.(56)-(58). We get then

$$
\begin{aligned}
q^{\lambda} T_{\lambda \mu \nu}^{A \rightarrow V V} & =-2 m i T_{\mu \nu}^{P \rightarrow V V}-\frac{(a-b)}{4 \pi^{2}} i \varepsilon_{\mu \nu \xi \eta} p^{\prime \eta} p^{\xi} \\
p^{\mu} T_{\lambda \mu \nu}^{A \rightarrow V V} & =-\frac{(b-a+2)}{8 \pi^{2}} i \varepsilon_{\lambda \nu \xi \eta} p^{\prime \eta} p^{\xi} \\
p^{\prime \nu} T_{\lambda \mu \nu}^{A \rightarrow V V} & =\frac{(b-a+2)}{8 \pi^{2}} i \varepsilon_{\lambda \mu \xi \eta} p^{\prime \eta} p^{\xi}
\end{aligned}
$$

Finally, we choose the value $a=1$ in the above expression to get

$$
\begin{aligned}
q^{\lambda} T_{\lambda \mu \nu}^{A \rightarrow V V} & =-2 m i T_{\mu \nu}^{P \rightarrow V V}-\frac{(1-b)}{4 \pi^{2}} i \varepsilon_{\mu v \xi \eta} p^{\prime \eta} p^{\xi} \\
p^{\mu} T_{\lambda \mu \nu}^{A \rightarrow V V} & =-\frac{(1+b)}{8 \pi^{2}} i \varepsilon_{\lambda v \xi \eta} p^{\prime \eta} p^{\xi} \\
p^{\prime \nu} T_{\lambda \mu \nu}^{A \rightarrow V V} & =\frac{(1+b)}{8 \pi^{2}} i \varepsilon_{\lambda \mu \xi \eta} p^{\prime \eta} p^{\xi}
\end{aligned}
$$

The result this way obtained, can be immediately recognized as the traditional one [2],[10], [11], [12]. It is now clear that there is no value for the $b$ parameter in order to preserve all Ward identities. Following the usual arguments and choosing the value $b=-1$ the $U(1)$ gauge symmetry is maintained, but the axial one is violated. Which have become clear in the discussion above is that the sources of the violating terms as well as of the anomalous term are $A V$ two-point function structures.

\section{FINAL REMARKS AND CONCLUSIONS}

Considering the facts stated in the last two sections we, apparently, have created a problematic situation if we want to look at all the problems on the same way, maintaining all the physical constraints simultaneously. The results produced by our treatment preserve all the arbitrariness intrinsic to the problem. All the considered Ward identities are put in terms of the same condition. So, the question is to choose what we need to choose in order to get the desirable consistency. The arbitrariness, however, can be related to the $S V$ twopoint function which is related to the $A V$ amplitude in a nonambiguous way. Due to physical reasons, and also in the DR, the $S V$ amplitude must be identically vanishing as well as the $A V$ one. The complex scalar field Ward identity must be preserved, as well as the two vector currents must be conserved in the $S V V$ process. Undoubtedly, only the choice $\Delta_{\mu \nu}^{r e g}=0$ can fulfill all these requirements. However, this choice eliminates 
all the ambiguities in the $A V V$ amplitude which are the ingredients usually used to justify the anomaly involved, and, apparently, forbids any violation of symmetry relations for this amplitude. How can we reconciliate this situation? In order to answer this question it is necessary to assume a conceptual and philosophical point of view for the problem: the traditional way used to justify the $A V V$ triangle anomaly cannot be maintained if we want to look at all the problems in the same way and the ambiguities cannot play a relevant role in a consistent interpretation of the perturbative amplitudes. In this line of reasoning we first need to break fundamental symmetries and general principles of QFT, by assuming the $S V$ and $A V$ structures as non-vanishing ones, for only after this get a justification for the perturbative origin of the anomalies. A powerful philosophical argument can be added to these objective ones. The $A V V$ anomaly phenomenon is predicted for the exact amplitudes, i.e., it is a fundamental phenomenon. However, in the eventual exact solutions for the corresponding QFT's equations of motion certainly the infinities and the associated ambiguities must be absent. So, we cannot expect that the justification of the origin of the anomaly phenomenon, even in perturbative solutions, resides in exclusive ingredients of the perturbative calculations as the infinities and ambiguities are. Given this argument, the answer for the question put above is intrinsically contained in the problem. Being a fundamental and unavoidable phenomenon, the anomaly should be present in any explicit expression for the $A V V$ amplitude. This means that no choices for the arbitrariness can eliminate the anomaly as well as no regularization prescription or equivalent philosophy. Then we can expect that a point of view for the anomalies can be constructed in accordance with all the others in perturbative calculations. For this purposes it is necessary to evaluate explicitly the $A V V$ and $P V V$ ampli- tudes imposing the consistency condition, and expecting that the violations emerge in a natural way. Which have become clear in the discussions presented here is that in the context of traditional regularization procedures different prescriptions are used for the treatment of identical mathematical structures depending on the context they appear.

The present status of the problem can be summarized as follows. In the situations where the DR can be used, eliminating the ambiguities, we certainly adopt it. In the situations where the involving mathematical structures are not naturally extendable to any dimension, which is the case of triangle anomalies, we adopt the surface's terms evaluation, attributing a meaning to the ambiguous character of the perturbative amplitudes. In a certain way, in situations where these problems do not simultaneously occur this option represents a possible choice for the involved arbitrariness. However, admitting the intention of looking at all the fundamental interactions as parts of a more general and unified theory, it seems a patently absurd idea because this means that in a certain amplitude of the same theory a value is attributed for the objects $\triangle_{\mu v}$, having in mind consistency reasons, while in other amplitudes the value can be taken as different without any crisis of conscience. Certainly it would be very frustrating for any physicist who got interested in studying an exact science to accept this situation as a final one. This situation is clearly unacceptable and additional efforts in order to achieve consistent and universal interpretations for the mathematical indefinitions intrinsic of the perturbative calculations are required. The strategy described in the section $\mathrm{V}$ seems to put the analysis in the right direction.

Acknowledgements: G.D. and O.A.B acknowledges a grant from $\mathrm{CNPq} / \mathrm{Brazil}$. E.G. acknowledges a grant from CAPES/Brazil.
[1] G.'t Hooft and M. Veltman, Nucl. Phys. B44 189 (1972); C.G. Bollini and J.J. Giambiagi, Phys. Lett. B40 566 (1972); J.F. Ashmore, Nuovo Cimento Lett. 4289 (1972).

[2] J. S. Bell and R. Jackiw, Nuovo Cim. A60, 47 (1969).

[3] S. Adler, Phys. Rev. 177, 2426 (1969).

[4] S. Adler and W.A. Bardeen, Phys. Rev. 182, 1517 (1969); W.A. Bardeen, Phys. Rev. 184, 1848 (1969).

[5] I.S. Gerstein and R. Jackiw, Phys. Rev. 181, 1955 (1969).

[6] V. Elias, Gerry McKeon, R.B. Mann, Nucl. Phys. B229 487 (1983).

[7] O.A. Battistel, PhD Thesis (1999), Universidade Federal de Minas Gerais, Brazil.
[8] O.A. Battistel and G. Dallabona, Phys. Rev. D65, 125017 (2002).

[9] O.A. Battistel and O.L. Battistel, Int. J. Mod. Phys. A17, 19792017 (2002).

[10] T.P. Cheng and L.F. Li, Gauge Theory of Elementary Particle Physics, Oxford University Press, New York (1984).

[11] P.H. Frampton, Gauge Field Theories Benjamin /Cummings, Menlo Park, California (1987).

[12] M. Kaku, Quantum Field Theory (Oxford University Press) (1993). 